\title{
Clinical evaluation of left ventricular function and morphology using an accelerated k-t sensitivity encoding method in cardiovascular magnetic resonance
}

Antonildes Nascimento Assuncao- $\mathrm{Jr}^{1 \dagger}$, Roberto Nery Dantas-Jr $\mathrm{Jr}^{1 * \dagger}$, Renata Margarida do Val ${ }^{1}$, Priscilla Gianotto ${ }^{1}$, Angela dos Santos Marin², Mark Golden, Marco Antonio Gutierrez ${ }^{1}$, Jose Rodrigues Parga ${ }^{1}$ and Cesar Higa Nomura'

\begin{abstract}
Objectives: To provide clinical validation of a recent 2D SENSE-based accelerated cardiovascular magnetic resonance (CMR) sequence (accelerated $k$-t SENSE), investigating whether this technique accurately quantifies left ventricle (LV) volumes, function, and mass as compared to 2D cine steady-state free precession (2D-SSFP).

Methods: Healthy volunteers $(n=16)$ and consecutive heart failure patients $(n=26)$ were scanned using a 1.5T MRI system. Two LV short axis (SA) stacks were acquired: (1) accelerated k-t SENSE (5-6 breath-holds; temporal/ spatial resolution: $37 \mathrm{~ms} / 1.82 \times 1.87 \mathrm{~mm}$; acceleration factor $=4)$ and $(2)$ standard 2D-SSFP (10-12 breath-holds; temporal/spatial resolution: $49 \mathrm{~ms} / 1.67 \times 1.87 \mathrm{~mm}$, parallel imaging). Ascending aorta phase-contrast was performed on all volunteers as a reference to compare LV stroke volumes (LVSV) and validate the sequences. An image quality score for SA images was used, with lower scores indicating better quality (from 0 to 18).
\end{abstract}

Results: There was a high agreement between accelerated k-t SENSE and 2D-SSFP for LV measurements: bias (limits of agreement) of $2.4 \%$ ( $-5.4 \%$ to $10.1 \%), 6.9 \mathrm{~mL} / \mathrm{m}^{2}\left(-4.7\right.$ to $\left.18.6 \mathrm{~mL} / \mathrm{m}^{2}\right),-1.5\left(-8.3\right.$ to $\left.5.2 \mathrm{~mL} / \mathrm{m}^{2}\right)$, and $-0.2 \mathrm{~g} / \mathrm{m}^{2}(-11.9$ to $12.3 \mathrm{~g} / \mathrm{m}^{2}$ ) for LV ejection fraction, end-diastolic volume index, end-systolic volume index, and mass index, respectively. LVSV by accelerated k-t SENSE presented good agreement with aortic flow. Interobserver and intraobserver variabilities for all LV parameters were also high.

Conclusion: The accelerated k-t SENSE CMR sequence is clinically feasible and accurately quantifies LV volumes, function, and mass, with short acquisition time and good image quality.

Keywords: Cardiac function test, Cine magnetic resonance imaging, Cardiac imaging techniques, Congestive cardiomyopathies, Ventricular ejection fraction

\section{Key points}

- CMR is considered the non-invasive gold standard method for ventricular functional measurements, accomplished mainly through 2D-SSFP cine images

* Correspondence: nerydjr@hotmail.com

Antonildes N. Assuncao-Jr and Roberto N. Dantas Jr are joint first authors.

${ }^{+}$Antonildes N. Assuncao-Jr and Roberto N. Dantas Jr contributed equally to this work.

${ }^{1}$ Heart Institute (InCor), University of Sao Paulo Medical School, Sao Paulo, Brazil

Full list of author information is available at the end of the article
- Accelerated imaging may favor patients with impaired breath-hold or frequent arrhythmia

- Recently developed accelerated CMR cine sequences speed up image acquisition either in temporal, spatial (k-space), or even both domains simultaneously (accelerated k-t SENSE)

- The accelerated $k$-t SENSE CMR sequence is clinically feasible and accurate for ventricular functional assessment, with short acquisition time and good image quality 


\section{Introduction}

Cardiovascular magnetic resonance (CMR) imaging is the non-invasive gold standard modality for quantification of left ventricle (LV) function, volumes, and mass [1]. For these purposes, a 2D cine steady-state free precession (2D-SSFP)-based sequence has been widely used in clinical practice, with high reproducibility and accuracy [1-3]. However, 2D-SSFP requires multiple breath-holds, resulting in prolonged exam duration, and representing a challenge for patients with heart failure symptoms. In addition to that, slice misregistration can be a result of inconsistent breath holding, hampering volumetric estimation [4-6]. Thus, the chosen imaging sequence for determined populations is crucial for delivering fast and accurate diagnostic information. In response to these issues, there have been considerable attempts to accelerate cine sequences, either in temporal, spatial (k-space), or even both domains simultaneously, without considerably reducing spatial or temporal resolutions [7-9].

Accelerated CMR sequences, such as k-t BLAST (broad-use linear acquisition speed-up technique; single receiver coil) and $\mathrm{k}-\mathrm{t}$ SENSE (sensitivity encoding; multiple receiver coils) consider spatiotemporal correlations throughout the image and rely on undersampling and signal overlap (aliasing) recovery through computational algorithms [7, 8]. Signal correlations and coil sensitivity estimates are obtained in an initial low-resolution acquisition (training stage), and this information is used afterwards for image reconstruction, allowing acceleration by sparsely sampling k-space over time in the main acquisition stage [9].

Recently, a new accelerated 2D k-t SENSE-based cine sequence (accelerated $\mathrm{k}-\mathrm{t}$ SENSE) was developed to accelerate image acquisition, without performing the training stage [10]. Signal correlations and coil sensitivity estimates are extracted from the acquisition stage data itself during post processing, allowing faster image acquisition [10]. Our goal in the present study is to clinically investigate whether this newly developed accelerated k-t SENSE sequence accurately quantifies LV volumes, function, and mass as compared to the 2D-SSFP cine with acceptable image quality.

\section{Materials and methods}

\section{Subjects}

Healthy volunteers $(n=16)$ and consecutive heart failure patients $(n=26)$ were prospectively enrolled in this study. Exclusion criteria were age under 18 years old, contraindications to CMR (e.g., CMR-incompatible devices, metallic bodies in the eye, intracranial metal clips), irregular heart rhythms, severely impaired breathhold capacity, claustrophobia, and pregnancy. All patients were clinically referred to CMR assessment of LV volumes, function, and mass. Indications for CMR in patients included non-ischemic $(n=20)$ and ischemic cardiomyopathies $(n=6)$. This study was approved by the Institutional Ethics Committee and all subjects provided written informed consent.

\section{Accelerated k-t SENSE technique}

The rationale and technical details of the acceleration sequence have been described elsewhere [10]. Briefly, accelerated k-t SENSE consists of a k-t SENSE-based SSFP cine sequence that uses no training stage (a previous additional low resolution scan used only for obtaining reference signals) for defining spatiotemporal signal correlations and $\mathrm{k}-\mathrm{t}$ coil sensitivity maps, thus allowing several fold acceleration. These signal correlations and sensitivity maps are estimated from the acquired image itself during post-processing. As a result, image acquisition is accelerated through undersampling of k-space over time in the main acquisition stage, which provides all correlations needed for final image reconstruction. Therefore, acceleration occurs in both spatial and temporal directions, with partially sampled data, allowing optimization of scan timing.

\section{MRI protocol}

All subjects were prospectively scanned using a 1.5T MRI system (Canon Vantage Titan, Canon Medical System Corporation, Japan). In addition to the standard protocol regarding the clinical indication, two short axis (SA) stacks fully covering both ventricles were acquired and their duration was measured: prospective ECG-triggered accelerated k-t SENSE cine (two slices/breath-hold), and a standard 2D-SSFP cine with parallel imaging and retrospective ECG triggering (one slice/breath-hold).

In order to validate the left ventricle stroke volume (LVSV) obtained by accelerated k-t SENSE, a retrospective phase-contrast flow measurement in the ascending aorta (immediately above sinotubular junction) was performed as a reference in all healthy subjects (VENC $200 \mathrm{~cm} / \mathrm{s}$, matrix $256 \times 88$, temporal/spatial resolution $46 \mathrm{~ms} / 1.48 \times$ $4.31 \mathrm{~mm})$. All cine sequences included standard shimming $(64 \times 64 \mathrm{~mm}$ matrix, voxel size $6.25 \times 6.25 \mathrm{~mm})$ and use of view sharing. Imaging parameters are described in Table 1.

\section{Assessment of LV function and morphology}

First, all images were de-identified and digitally stored. Then, for quantitative measurements, two experienced cardiovascular imaging specialists ( $>5$ years of training) analyzed the SA stacks-twice by one reader (time interval between readings of 1 week), using a commercially available software Medis Suite 3.0 (Medis, Leiden, The Netherlands).

All endocardial and epicardial contours were manually drawn as recommended by the Society for Cardiovascular Magnetic Resonance Guidelines [11], covering LV 
Table 1 Imaging parameters

\begin{tabular}{lll}
\hline & 2D-SSFP & Accelerated k-t SENSE \\
\hline ECG triggering & Retrospective & Prospective \\
TE/TR (ms) & $1.7 / 3.4$ & $1.7 / 3.4$ \\
FOV (mm) & $320 \times 360$ & $320 \times 360$ \\
Image matrix & $192 \times 192$ & $176 \times 192$ \\
Spatial resolution (mm) & $1.67 \times 1.87$ & $1.82 \times 1.87$ \\
Temporal resolution (ms) & 49 & 37 \\
Slice thickness/spacing (mm) & $10 / 0$ & $10 / 0$ \\
Flip angle $\left(^{\circ}\right.$ ) & 60 & 60 \\
Bandwidth (Hz/pixel) & 977 & 1302 \\
Cardiac phases ( $n$ ) & 25 & 32 \\
View sharing (segments) & 14 & 11 \\
Breath-holds ( $n$ ) & $10-12$ & $5-6$ \\
Slices per breath-hold & 1 & 2 \\
Acceleration factor & Parallel imaging & $4 \times$ \\
Acquisition duration (s) & $161 \pm 25$ & $67 \pm 14$ \\
\hline
\end{tabular}

TE echo time, TR repetition time, FOV field of view, 2D-SSFP 2D steady-state free precession

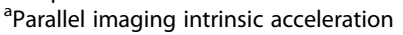

from basal to apical slices, including both papillary muscles and trabeculations as part of the LV cavity. For basal slices, contour was carefully drawn to include the LV outflow tract to the level of the aortic valve cusps, and left atrium was recognized when less than $50 \%$ of the blood volume was surrounded by ventricular myocardium [11].

LV volumes (end-diastolic-LVEDV and end-systolicLVESV), ejection fraction (LVEF), and mass, and their respective indexes corrected for the body surface area, were calculated using the Simpson method. End-diastole phases were chosen as those with the maximum volume in a mid-ventricular slice to provide more consistent estimations of the LV volumes [12]. Quantitative wall motion per-segment analysis (American Heart Association-16-segment model) was also performed, using a centerline method applied to the endocardial and epicardial contours at end-diastole and end-systole [13].

For aortic flow quantification on the healthy volunteers group, the borders of the ascending aorta were traced to include only its cavity. For optimal results, image plane was properly centered and aligned. Aliasing was double checked and, if occurred, velocity encoding sensitivity was set accordingly. The final aortic flow included the estimated coronary arteries flow (mL/beat), calculated as $0.8 \times \mathrm{LV}$ mass (g) / heart rate (beats/min) [14].

\section{Image quality}

Quality assessment of cine CMR SA images was performed based on 11 qualitative criteria [15]. This assessment yields a score for LV coverage, presence of artifacts (wrap around, ghosts, metallic and shimming artifacts, image blurring/mis-triggering), signal loss, correct orientation of stack, and adequate gap between slices, assigning individual scores that range from 0 to 18 (the lower the score, the better the image quality) (Table 2).

\section{Statistical analysis}

Continuous variables are shown as mean \pm standard deviation, and were compared using paired $t$ test or paired Wilcoxon test, as appropriate. Categorical variables were expressed as frequencies (percentages). Agreement of LV parameters on the accelerated $k-t$ SENSE and 2D-SSFP as well as agreement between volumetrically determined LVSV and aortic flow were assessed by Bland-Altman analysis. The same analysis was performed to investigate interobserver and intraobserver agreement. Repeatability

Table 2 Image quality of accelerated k-t SENSE and 2D-SSFP method

\begin{tabular}{|c|c|c|c|c|c|c|c|c|}
\hline & \multicolumn{4}{|l|}{ 2D-SSFP } & \multicolumn{4}{|l|}{$k-t$} \\
\hline & Score 0 & Score 1 & Score 2 & Score 3 & Score 0 & Score 1 & Score 2 & Score 3 \\
\hline Image blurring/mis triggering & 35 & 5 & 2 & 0 & 30 & 5 & 5 & 2 \\
\hline Shimming & 40 & 1 & 1 & 0 & 37 & 0 & 2 & 3 \\
\hline Ghosts & 42 & 0 & 0 & 0 & 36 & 6 & 0 & 0 \\
\hline \multicolumn{9}{|l|}{ Correct LV long axes } \\
\hline LV coverage & 42 & 0 & 0 & 0 & 42 & 0 & 0 & 0 \\
\hline Metallic artifact & 42 & 0 & 0 & 0 & 42 & 0 & 0 & 0 \\
\hline Orientation of stack & 42 & 0 & 0 & 0 & 42 & 0 & 0 & 0 \\
\hline Signal loss & 42 & 0 & 0 & 0 & 42 & 0 & 0 & 0 \\
\hline Slice thickness/Gap & 42 & 0 & 0 & 0 & 42 & 0 & 0 & 0 \\
\hline Wrap around & 42 & 0 & 0 & 0 & 42 & 0 & 0 & 0 \\
\hline Total score ${ }^{a}$ & \multicolumn{4}{|c|}{$0.33 \pm 0.64$} & \multicolumn{4}{|c|}{$0.98 \pm 1.25$} \\
\hline
\end{tabular}

2D-SSFP 2D steady-state free precession, $L V$ left ventricle

${ }^{a}$ mean $\pm S D$ 
Table 3 Study population

\begin{tabular}{|c|c|c|c|}
\hline & Patients $(n=26)$ & Volunteers $(n=16)$ & $p$ value \\
\hline \multicolumn{4}{|l|}{ Demographics } \\
\hline Age, years & $53 \pm 13$ & $43 \pm 14$ & $<0.001$ \\
\hline Male, $n(\%)$ & $17(65)$ & $10(63)$ & 0.91 \\
\hline $\mathrm{BMI}, \mathrm{kg} / \mathrm{m}^{2}$ & $29 \pm 6$ & $26 \pm 3$ & 0.004 \\
\hline BSA, $\mathrm{m}^{2}$ & $1.91 \pm 0.27$ & $1.83 \pm 0.24$ & 0.33 \\
\hline $\mathrm{HR}, \mathrm{bpm}$ & $68 \pm 17$ & $63 \pm 17$ & 0.73 \\
\hline PVC, $n(\%)$ & $1(4 \%)$ & - & - \\
\hline \multicolumn{4}{|l|}{ Cardiovascular risk factors } \\
\hline Hypertension, n (\%) & $17(65)$ & $1(6)$ & - \\
\hline Diabetes, $n(\%)$ & $8(31)$ & - & - \\
\hline Hypercholesterolemia, n (\%) & $11(42)$ & - & - \\
\hline Smoking, n (\%) & $10(39)$ & - & - \\
\hline \multicolumn{4}{|l|}{ Diagnosis } \\
\hline HCM, n (\%) & $7(27)$ & - & - \\
\hline Ischemic heart disease, $n(\%)$ & $6(24)$ & - & - \\
\hline Chagas heart disease, $n(\%)$ & $3(11)$ & - & - \\
\hline DCM, $n(\%)$ & $3(11)$ & - & - \\
\hline Other cardiomyopathies, $n$ (\%) & $7(27)$ & - & - \\
\hline \multicolumn{4}{|l|}{$C M R$ findings ${ }^{a}$} \\
\hline LVEDVI, mL/m² & $92 \pm 50$ & $66 \pm 10$ & 0.01 \\
\hline LVESVI, mL/m² & $52 \pm 53$ & $26 \pm 10$ & 0.04 \\
\hline LVMI, $\mathrm{g} / \mathrm{m}^{2}$ & $80 \pm 28$ & $54 \pm 10$ & $<0.001$ \\
\hline LVSV, mL & $76 \pm 25$ & $75 \pm 15$ & 0.90 \\
\hline LVEF, \% & $52 \pm 21$ & $62 \pm 5$ & 0.05 \\
\hline
\end{tabular}

$B M I$ body index mass, $B S A$ body surface area, $H R$ heart rate, $P V C$ premature ventricular contraction, $H C M$ hypertrophic cardiomyopathy, DCM dilated cardiomyopathy, CMR cardiovascular magnetic resonance, LVEDVI left ventricle end-diastolic volume index, LVESVI left ventricle end-systolic volume index, LVMI left ventricle mass index, LVSV left ventricle stroke volume, LVEF left ventricle ejection fraction

Plus-minus values are means $\pm S D$

aLV measurements obtained from 2D-SSFP cine
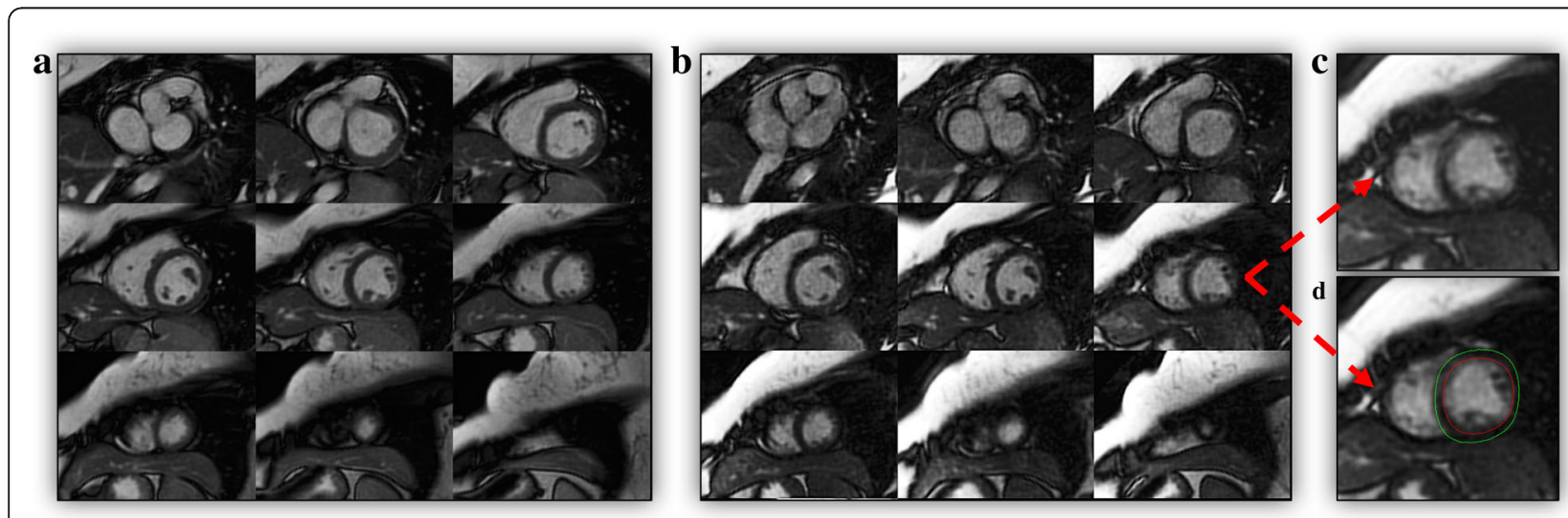

Fig. 1 Short-axis images acquired using 2D-SSDFP (a) and accelerated k-t SENSE sequence (b). Presence of blurring/mis-triggering (c) in LV apical slices of accelerated k-t SENSE cine and endocardial/epicardial contours manually corrected (d) 
$\mathbf{a}$

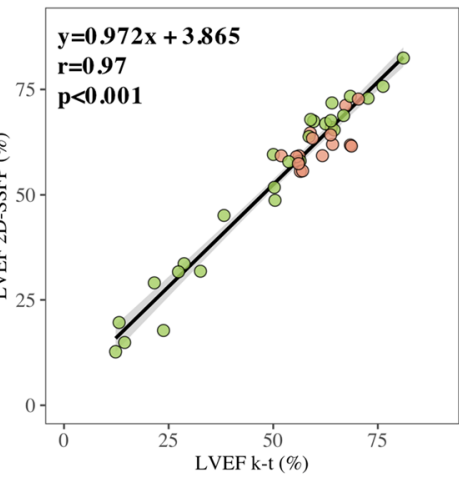

c

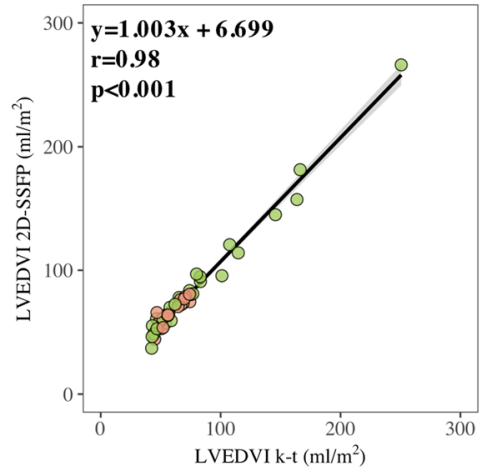

e

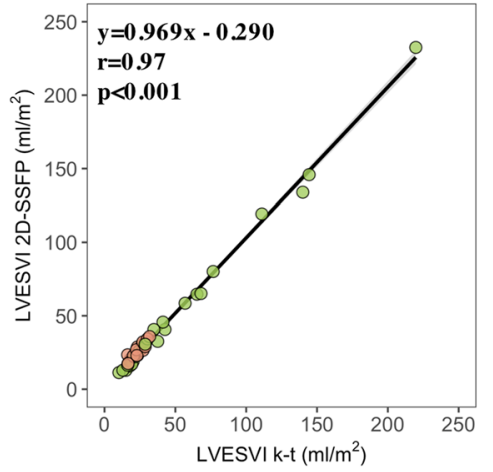

g

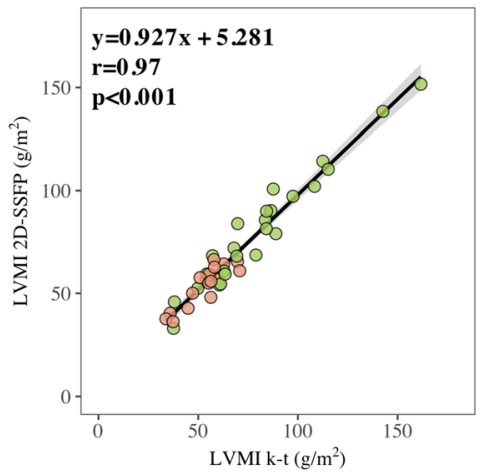

b

O Patients $\bigcirc$ Volunteers

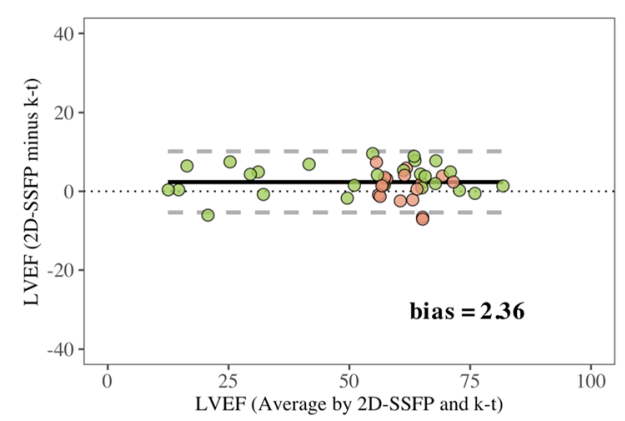

d

O Patients O Volunteers

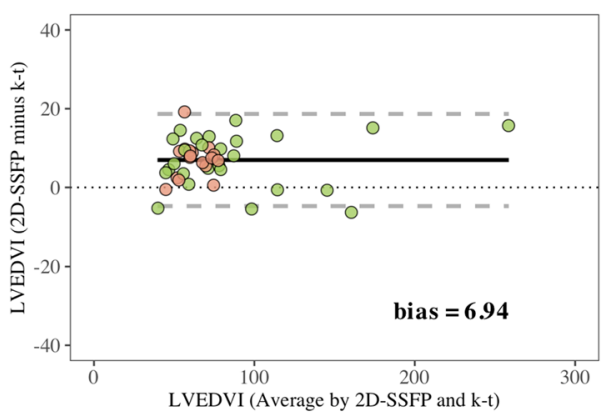

f

Patients $\bigcirc$ Volunteers

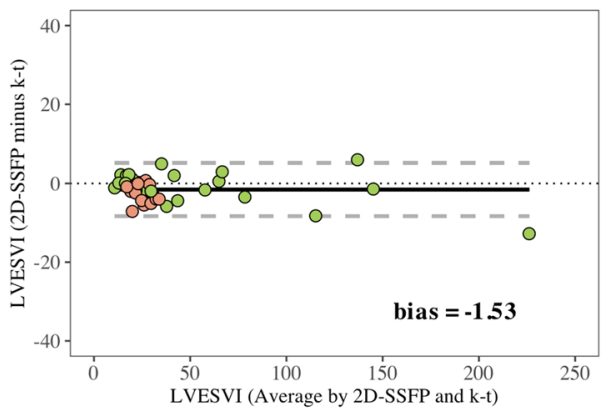

h

$\bigcirc$ Patients $\bigcirc$ Volunteers

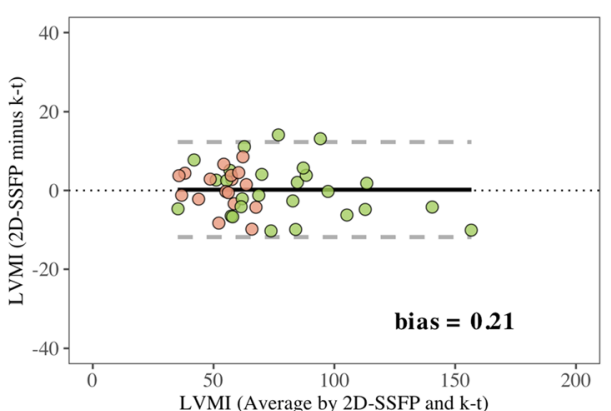

Fig. $\mathbf{2}$ LV volumes, function and mass by accelerated k-t SENSE and 2D-SSFP images. Correlations $(\mathbf{a}, \mathbf{c}, \mathbf{e}, \mathbf{g})$ and Bland-Altman analysis (b, d $, \mathbf{f}, \mathbf{h})$. LVEF left ventricular ejection fraction, LVEDVI left ventricular end-diastolic volume index, LVESVI left ventricular end-systolic volume index, LVMI left ventricular mass index 
coefficients (RC), two times the SDs of the differences between the two measurements, were also calculated for each LV parameter. All statistical analyses were performed using the software R 3.4.3 (The R Foundation for Statistical Computing, Vienna, Austria) and a $p$ value $<0.05$ was considered statistically significant.

\section{Results}

Study population, acquisition duration, and image quality Characteristics of the study population are displayed in Table 3. Most patients were referred to investigate non-ischemic cardiomyopathies and presented mean LVEF of $52 \pm 21 \%(p=0.05)$, with larger LV volumes than volunteers $(p$ values $<0.05)$. One patient presented infrequent premature ventricular contractions and all individuals had sinus rhythm. The accelerated k-t SENSE reduced by nearly $60 \%$ acquisition duration of short-axis images compared to $2 \mathrm{D}-\mathrm{SSFP}(67 \pm 14 \mathrm{~s}$ versus $161 \pm 25 \mathrm{~s}$, $p<0.001$ ), with less breath-holds (Table 1).

Mean quality score resulted in high image quality for both methods, yet 2D-SSFP performed slightly better $(0.33 \pm 0.64$ versus $0.98 \pm 1.25, p<0.001)$ (Table 2). Five accelerated k-t SENSE acquisitions (12\%) had score $\geq 3$ due to either image blurring/mis-triggering or shimming artifacts (Table 2), but they did not preclude adequate recognition of endocardial/epicardial borders (Fig. 1).

\section{Agreement between accelerated k-t SENSE and 2D-SSFP cine}

There was a strong correlation between accelerated k-t SENSE and 2D-SSFP for quantification of LV measurements, with high agreement. Mean difference or bias (limits of agreement, LOA) were $2.4 \%$ (- $5.4 \%$ to $10.1 \%)$, $6.9 \mathrm{~mL} / \mathrm{m}^{2}$ ( -4.7 to $\left.18.6 \mathrm{~mL} / \mathrm{m}^{2}\right),-1.5(-8.3$ to $5.2 \mathrm{~mL} /$ $\mathrm{m}^{2}$ ), and $-0.2 \mathrm{~g} / \mathrm{m}^{2}$ (LOA -11.9 to $12.3 \mathrm{~g} / \mathrm{m}^{2}$ ) for LVEF, left ventricle end-diastolic volume index (LVEDVI), left ventricle end-systolic volume index (LVESVI), and left ventricle mass index (LVMI), respectively (Fig. 2). Regional myocardial wall motion analysis also showed good correlation $(0.63 \leq$ correlation coefficient $\leq 0.87$, all $p$ values <0.001) and agreement $(-0.74 \leq$ bias $\leq 0.06)$ between accelerated k-t SENSE and 2D-SSFP (Fig. 3). Interobserver and intraobserver variabilities of accelerated $\mathrm{k}-\mathrm{t}$ SENSE ranged from $0.1 \mathrm{~mL} / \mathrm{m}^{2}$ to $-5.2 \mathrm{~g} /$ $\mathrm{m}^{2}$ and from $0.6 \mathrm{~mL} / \mathrm{m}^{2}$ to $-4.5 \mathrm{~g} / \mathrm{m}^{2}$, respectively for LVEDVI and LVMI. RC ranged from 6\% (LVEF) to $14 \mathrm{~g} / \mathrm{m}^{2}$ (LVMI) (Table 4 ).

\section{Validation of accelerated k-t SENSE against aortic flow}

The quantification of LVSV by the accelerated k-t SENSE showed a strong correlation and good agreement with aortic flow, slightly underestimating LVSV by $0.58 \mathrm{~mL}$ (Fig. 4).

\section{Discussion}

In this study, the accelerated k-t SENSE cine (no training stage approach) was compared to 2D-SSFP for the quantification of LV measurements, with high agreement. The accelerated sequence required half the breath-holds (5 to 6 vs 10 to 12) to cover the entire LV (reducing acquisition time by $60 \%$ ), and yielded an excellent image quality in $88 \%$ of subjects, with accurate assessment of LV function, volumes, and mass as compared to the 2D-SSFP cine.
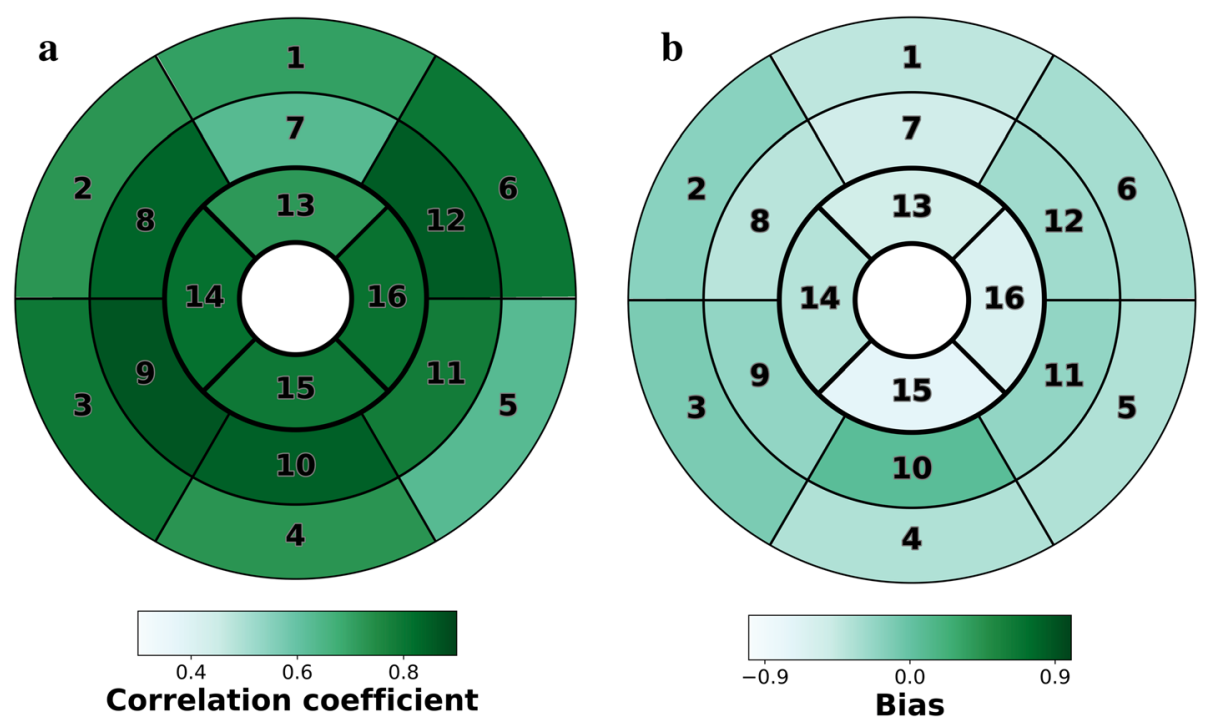

Fig. 3 Quantitative regional wall motion correlation and agreement between accelerated k-t SENSE and 2D-SSFP images. 16-Segment American Heart Association bullseye plots indicate the correlation coefficients (a) and mean differences or bias (b) (in mm) for wall motion assessed by accelerated $k$-t SENSE and 2D-SSFP cine images 
Table 4 Interobserver and intraobserver reproducibility of accelerated k-t SENSE

\begin{tabular}{|c|c|c|c|c|c|c|}
\hline & \multicolumn{3}{|l|}{ Interobserver } & \multicolumn{3}{|l|}{ Intraobserver } \\
\hline & Agreement bias (LOA) & Correlation $r, p$ value & $\mathrm{RC}$ & Agreement bias ( $L O A)$ & Correlation $r, p$ value & $\mathrm{RC}$ \\
\hline LVEF, \% & $-2.6(-8.2$ to 3.6$)$ & $0.98, p<0.001$ & 6 & $1.7(-6.5$ to 9.8$)$ & $0.96, p<0.001$ & 8 \\
\hline LVEDVI, $\mathrm{mL} / \mathrm{m}^{2}$ & $0.1(-12.2$ to 12.2$)$ & $0.98, p<0.001$ & 12 & $0.6(-10.8$ to 12.1$)$ & $0.95, p<0.001$ & 12 \\
\hline LVESVI, mL/m² & $2.2(-7.7$ to 9.9$)$ & $0.98, p<0.001$ & 10 & $0.7(-9.9$ to 8.5$)$ & $0.98, p<0.001$ & 9 \\
\hline LVMI, g/m² & $-5.2(-19.3$ to 8.9$)$ & $0.95, p<0.001$ & 14 & $-4.5(-16.5$ to 7.5$)$ & $0.98, p<0.001$ & 12 \\
\hline
\end{tabular}

$\angle O A$ limits of agreement, $R C$ repeatability coefficient, $L V E F$ left ventricle ejection fraction, LVEDVI left ventricle end-diastolic volume index, $L V E S V I$ left ventricle endsystolic volume index, $L V M I$ left ventricle mass index

Agreement between accelerated and standard cines was high for LVEF, with only a slightly wider variability $(-4.1 \%$ to $4.3 \%)[3,16]$. Additionally, accelerated k-t SENSE was quite robust regarding reproducibility for LVEF (RC 6\% and $8 \%$ for inter- and intraobserver, respectively), closely matching the interobserver reproducibility of 2D-SSFP cine $(6 \%$ and $12 \%)[17,18]$. Accelerated k-t SENSE images also provided comparable results in the quantitative regional myocardial wall motion analysis compared to 2D-SSFP.

Likewise, a relevant agreement between the techniques was achieved for LV volumes and mass. Accelerated k-t SENSE promoted a small myocardial mass overestimation and LVEDVI underestimation in a similar fashion of what was found when studying agreement of a 3D k-t BLAST technique with 2D-SSFP cines (despite differences between BLAST and SENSE based sequences) [8]. Other studies regarding spatiotemporal acceleration techniques found similar results, either with compressed sensing or SENSE $[19,20]$. Accelerated k-t SENSE had also high accuracy for calculating LVSV using as reference the aortic flow, with a very small overestimation of $0.58 \mathrm{~mL}$. This result is in line with the reported overestimation of LVSV by accelerated sequences in aortic valves [20,21].

Although the vast majority of patients had excellent image quality, accelerated k-t SENSE yielded, on average, a higher image quality score when compared to the $2 \mathrm{D}$
SSFP cine. Indeed, accelerated sequences may favor blurring or residual aliasing artifacts, degrading image quality $[22,23]$. However, even in patients with artifacts, images were reasonable for adequate recognition of myocardial borders.

It is remarkable that, despite not reducing acquisition time comparable to neither compressed sensing [20] nor whole heart 3D cine (about 80\%) [23], accelerated k-t SENSE reached $60 \%$ faster cine acquisition. However, these two other techniques present high computational burden for image reconstruction (precluding immediate assessment of quality or planning next steps) and reporting time (to up $30 \mathrm{~min}$ ), making accelerated k-t SENSE advantageous.

Some further limitations must be outlined. Firstly, although we included heart failure patients, they had no limiting symptoms and/or significant arrhythmias given the study design. Therefore, additional studies are planned to confirm whether this expressive acquisition time reduction also benefits subjects with more limited breath-hold capabilities. Secondly, despite the possibility of using a wider range of acceleration factors by the accelerated k-t SENSE, faster acquisition $(>4 \times)$ accuracy still needs evaluation regarding reliability, as higher acceleration factors with k-t SENSE produced poorer image quality and agreement in previous experience [9]. Finally, retrospective accelerated k-t SENSE was not fully
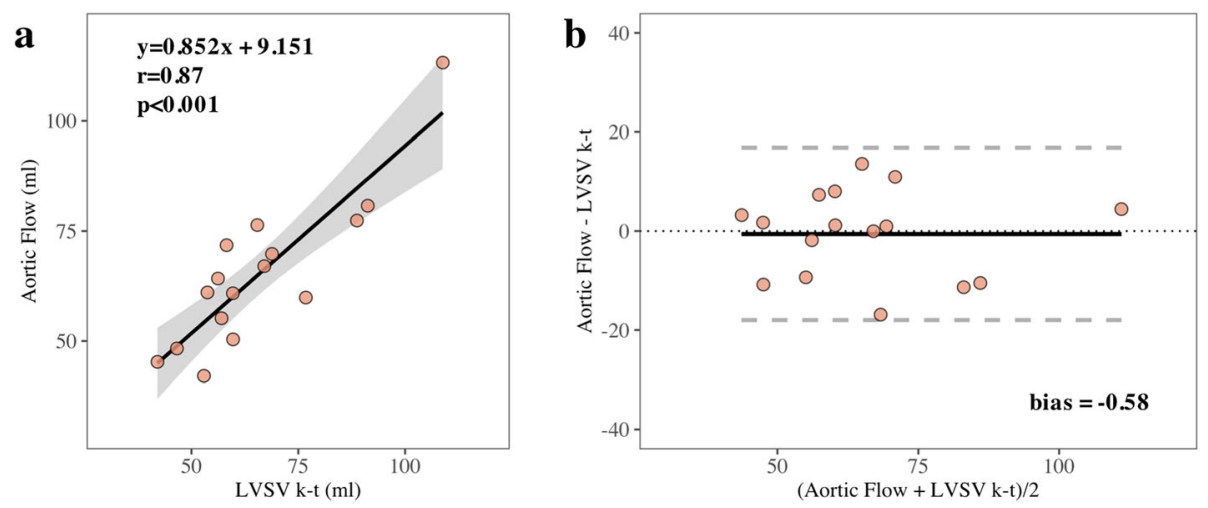

Fig. 4 Validation analysis for quantification of stroke volume by accelerated k-t SENSE versus aortic forward flow. Correlation (a) and Bland-Altman analysis (b). LVSV left ventricular stroke volume 
developed by the completion of this work, and may represent further achievements in function assessment.

In conclusion, the accelerated $k-t$ SENSE is feasible and can accurately quantify LV volumes, function, and mass, with good image quality and considerable shortening of acquisition time.

\section{Abbreviations}

2D-SSFP: 2D cine steady-state free precession; BLAST: Broad-use linear acquisition speed-up technique; BSA: Body surface area; CMR: Cardiovascular magnetic resonance; $L V$ : Left ventricle; LVEDVI: Left ventricle end-diastolic volume index; LVEF: Left ventricle ejection fraction; LVESVI: Left ventricle endsystolic volume index; LVMI: Left ventricle mass index; LVSV: Left ventricle stroke volume; NYHA: New York Heart Association; SA: Short axis;

SENSE: Sensitivity encoding

\section{Funding}

This work received research support from Canon Medical Systems do Brasil.

\section{Authors' contributions}

AA and RD idealized, carried out all stages of the study, and drafted the manuscript. JP, CN and MG participated inthe study design. AM participated in the sequence alignment. AA performed the statistical analysis. PG, AA and $\mathrm{RD}$ performed image acquisition and evaluation. RV participated in its design and coordination. CN and JP were the scientific guarantors of this publication. All authors read and approved the final manuscript.

\section{Competing interests}

The authors declare that they have no competing interests.

\section{Publisher's Note}

Springer Nature remains neutral with regard to jurisdictional claims in published maps and institutional affiliations.

\section{Author details}

'Heart Institute (InCor), University of Sao Paulo Medical School, Sao Paulo, Brazil. ${ }^{2}$ Canon Medical Systems do Brasil, Sao Paulo, Brazil. ${ }^{3}$ Canon Medical Systems Corporation, Otawara, Japan.

Received: 18 December 2018 Accepted: 13 May 2019

Published online: 13 June 2019

\section{References}

1. Bellenger N, Burgess MI, Ray SG et al (2000) Comparison of left ventricular ejection fraction and volumes in heart failure by echocardiography, radionuclide ventriculography and cardiovascular magnetic resonance. Are they interchangeable? Eur Heart J 21:1387-1396. https://doi.org/10.1053/ euhj.2000.2011

2. Lorenz CH, Walker ES, Morgan VL, Klein SS, Graham TP Jr (1999) Normal human right and left ventricular mass, systolic function, and gender differences by cine magnetic resonance imaging. J Cardiovasc Magn Reson $1: 7-21$

3. Grothues F, Smith GC, Moon JC et al (2002) Comparison of interstudy reproducibility of cardiovascular magnetic resonance with two-dimensional echocardiography in normal subjects and in patients with heart failure or left ventricular hypertrophy. Am J Cardiol 90:29-34

4. Jeong D, Schiebler ML, Lai P, Wang K, Vigen KK, François CJ (2015) Single breath hold 3D cardiac cine MRI using kat-ARC: preliminary results at 1.5T. Int J Cardiovasc Imaging 31:851-857. https://doi.org/10.1007/s10554-0150615-0

5. Chandler AG, Pinder RJ, Netsch T et al (2008) Correction of misaligned slices in multi-slice cardiovascular magnetic resonance using slice-tovolume registration. J Cardiovasc Magn Reson 10:13. https://doi.org/10. 1186/1532-429X-10-13

6. Huber S, Muthupillai R, Mojibian H, Cheong B, Kouwenhoven M, Flamm SD (2008) Rapid assessment of regional and global left ventricular function using three-dimensional k-t BLAST imaging. Magn Reson Imaging 26:727738. https://doi.org/10.1016/j.mri.2008.01.027
7. Tsao J, Boesiger P, Pruessmann KP (2003) K-t BLAST and k-t SENSE: dynamic MRI with high frame rate exploiting spatiotemporal correlations. Magn Reson Med 50:1031-1042. https://doi.org/10.1002/mrm.10611

8. Jaroni J, Meier R, Beer A et al (2013) Three-dimensional magnetic resonance imaging using single breath-hold k-t BLAST for assessment of global left ventricular functional parameters. Acad Radiol 20:987-994. https://doi.org/ 10.1016/j.acra.2013.03.012

9. Maredia N, Kozerke S, Larghat A et al (2008) Measurement of left ventricular dimensions with contrast-enhanced three-dimensional cine imaging facilitated by k-t SENSE. J Cardiovasc Magn Reson 10:27. https://doi.org/10. 1186/1532-429X-10-27

10. Takeshima H, Saitoh K, Nitta S et al (2019) Estimation of spatiotemporal sensitivity using band-limited signals with no additional acquisitions for $k-t$ parallel imaging. Magn Reson Med Sci 18:19-28. https://doi.org/10.2463/ mrms.mp.2017-0132

11. Schulz-Menger J, Bluemke DA, Bremerich J et al (2013) Standardized image interpretation and post processing in cardiovascular magnetic resonance: Society for Cardiovascular Magnetic Resonance (SCMR) board of trustees task force on standardized post processing. J Cardiovasc Magn Reson 15:35. https://doi.org/10.1186/1532-429X-15-35

12. Contijoch F, Witschey WR, Rogers K et al (2016) Impact of end-diastolic and end-systolic phase selection in the volumetric evaluation of cardiac MRI. J Magn Reson Imaging 43:585-593. https://doi.org/10.1002/jmri.25038

13. Holman ER, Buller VG, de Roos A et al (1997) Detection and quantification of dysfunctional myocardium by magnetic resonance imaging. A new three-dimensional method for quantitative wall-thickening analysis. Circulation 95:924-931

14. Schwitter J, DeMarco T, Kneifel $\mathrm{S}$ et al (2000) Magnetic resonance-based assessment of global coronary flow and flow reserve and its relation to left ventricular functional parameters : a comparison with positron emission tomography. Circulation 101:2696-2702. https://doi.org/10. 1161/01.CIR.101.23.2696

15. Klinke V, Muzzarelli S, Lauriers N et al (2013) Quality assessment of cardiovascular magnetic resonance in the setting of the European CMR registry: description and validation of standardized criteria. J Cardiovasc Magn Reson 15:55. https://doi.org/10.1186/1532-429X-15-55

16. Danilouchkine MG, Westenberg JJ, de Roos A, Reiber JH, Lelieveldt BP (2005) Operator induced variability in cardiovascular MR: left ventricular measurements and their reproducibility. J Cardiovasc Magn Reson 7:447-457

17. Papavassiliu T, Kühl HP, Schröder M et al (2005) Effect of endocardial trabeculae on left ventricular measurements and measurement reproducibility at cardiovascular MR imaging. Radiology 236:57-64. https:/ doi.org/10.1148/radiol.2353040601

18. Moon JC, Lorenz CH, Francis JM, Smith GC, Pennell DJ (2002) Breath-hold FLASH and FISP cardiovascular MR imaging: left ventricular volume differences and reproducibility. Radiology 223:789-797. https://doi.org/10. 1148/radiol.2233011181

19. Young AA, Cowan BR, Schoenburg SO, Wintersperger BJ (2008) Feasibility of single breath-hold left ventricular function with 3 Tesla TSENSE acquisition and 3D modeling analysis. J Cardiovasc Magn Reson 10:24. https://doi.org/ 10.1186/1532-429X-10-24

20. Vincenti G, Monney P, Chaptinel I et al (2014) Compressed sensing singlebreath-hold CMR for fast quantification of LV function, volumes, and mass. JACC Cardiovasc Imaging 7:882-892. https://doi.org/10.1016/j.jcmg.2014.04.016

21. Muzzarelli S, Monney P, O'Brien K et al (2014) Quantification of aortic flow by phase-contrast magnetic resonance in patients with bicuspid aortic valve. Eur Heart J Cardiovasc Imaging 15:77-84. https://doi.org/10. 1093/ehjci/jet129

22. Tsao J, Kozerke S, Boesiger P, Pruessmann KP (2005) Optimizing spatiotemporal sampling for $k$-t BLAST and k-t SENSE: application to highresolution real-time cardiac steady-state free precession. Magn Reson Med 53:1372-1382. https://doi.org/10.1002/mrm.20483

23. Maffei E, Messalli G, Martini C et al (2011) Magnetic resonance assessment of left ventricular volumes and mass using a single-breath-hold 3D k-t BLAST cine b-SSFP in comparison with multiple-breath-hold 2D cine b-SSFP. Insights Imaging 2:39-45. https://doi.org/10.1007/s13244-010-0056-1 